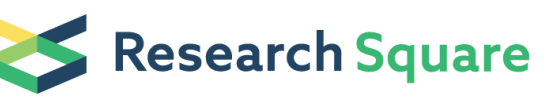

Preprints are preliminary reports that have not undergone peer review.

They should not be considered conclusive, used to inform clinical practice, or referenced by the media as validated information.

\section{Immunological Anti-Tumor Activity of Icariside I by Regulating Gut Microbiota and Its Derived Metabolites in a Melanoma Mouse Model}

\section{Gui Chen}

Wuhan Institute of Physics and Mathematics Chinese Academy of Sciences

\section{Zheng Cao}

Wuhan Institute of Physics and Mathematics Chinese Academy of Sciences

Zunji Shi ( $\square$ shizunji@mail.ccnu.edu.cn )

Wuhan Institute of Physics and Mathematics Chinese Academy of Sciences

Hehua Lei

Wuhan Institute of Physics and Mathematics Chinese Academy of Sciences

\section{Chuan Chen}

Wuhan Institute of Physics and Mathematics Chinese Academy of Sciences

\section{Peihong Yuan}

Wuhan Institute of Physics and Mathematics Chinese Academy of Sciences

\section{Fang Wu}

Wuhan Institute of Physics and Mathematics Chinese Academy of Sciences

Caixiang Liu

Wuhan Institute of Physics and Mathematics Chinese Academy of Sciences

\section{Manyuan Dong}

Wuhan Institute of Physics and Mathematics Chinese Academy of Sciences

\section{Yuchen Song}

Wuhan Institute of Physics and Mathematics Chinese Academy of Sciences

Jinlin Zhou

Golden Health(Guangdong) Biotechnology Co., Ltd

\section{Yujing Lu}

Guangdong University of Technology

\section{Limin Zhang ( $\nabla$ zhanglm@wipm.ac.cn )}

Wuhan Institute of Physics and Mathematics Chinese Academy of Sciences

\section{Research}

Keywords: Melanoma, Icariside I, Gut microbiota, Systematic immunity 
Posted Date: September 1st, 2020

DOl: https://doi.org/10.21203/rs.3.rs-66227/v1

License: (c) (i) This work is licensed under a Creative Commons Attribution 4.0 International License. Read Full License 


\section{Abstract \\ Background}

Emerging evidence has shown that gut microbiome plays important roles in antitumor immunotherapy by shaping systemic immune responses. Specifically, Lactobacillus spp. and Bifidobacterium spp. were previously reported to enhance host immunity and inhibit tumor growth. Furthermore, microbiota-derived metabolites such as short-chain fatty acids (SCFAs) and indole derivatives are gradually regarded as immunomodulatory molecules involved in metabolic signaling pathways.

\section{Methods}

A B16F10 melanoma model was selected as a tumor-bearing animal for the investigation of immunological anti-tumor activity of Icariside I. Gut microbiota structure and composition in cecal content of mice was firstly analyzed by 16S rRNA gene sequence with the goal of screening those bacteria highly associated with tumor immunotherapy. Subsequently, targeted metabolomics approach was employed to quantitatively determine microbiota-derived metabolites such as SCFAs and indole derivatives. The population of multiple lymphocyte subsets in peripheral blood were measured with flowcytometry to evaluate changes of host immunity.

\section{Results}

Icariside I significantly inhibited B16F10 melanoma growth in vivo through regulation of gut microbiota and host immune. Oral administration of icariside I improved the altered gut microbiota community with marked restoration of Lactobacillus spp. and Bifidobacterium spp. abundance. Icariside I was also able to improve the levels of microbiota-derived metabolites such as short-chain fatty acids (SCFAs) and indole derivatives, consequently repairing intestinal barrier and systemic inflammation of tumor-bearing mice. Furthermore, icariside I up-regulated multiple lymphocyte subsets including CD $4+$ and CD $8+T$ cells or NK and NKT cells in peripheral blood.

\section{Conclusions}

These results suggested that icariside I may be developed as a novel and natural anticancer adjuvant via microbiome remodeling and host immune regulation.

\section{Highlights}

Icariside I inhibits melanoma growth in B16F10-xenograft model.

Icariside I improves intestinal Lactobacillus spp.and Bifidobacterium spp. abundance. 
Icariside I modulates microbiota-derived metabolites (SCFAs and indole metabolites).

Icariside I enhances host immunity with elevation of multiple lymphocyte subsets.

\section{Background}

Melanoma is the most lethal form of skin cancer due to its high metastasis and drug resistance [1]. In the past, melanoma was mainly treated by surgery, radiotherapy and chemotherapy with short window period and high rate of recurrence [2]. In recent years, targeted therapeutic agents such as BRAF and MEK inhibitors or their combination were clinically applied for patients with metastatic melanoma and especially BRAF-mutant melanoma [3]. Currently, immunotherapy strategies have been developed from cytokine-based treatment to antibody-mediated blockade of the cytotoxic T-lymphocyte-associated antigen-4 (CTLA-4) and the programmed cell-death protein 1 (PD-1) immune checkpoints for melanoma patients. Although a variety of cancers such as melanoma and non-small-cell lung carcinoma have been approved by the FDA for immunotherapy with increasing the survival rate [4], there are still many patients who are ineffective or partially respond at immunotherapy [5]. Furthermore, antibodies targeting CTLA-4 and PD-1 for tumor immunotherapy are required for intravenous antibody delivery with possibility of tumor recurrence and side effects.

Increasing evidence has shown that the gut microbiome is closely related to multiple pathophysiological processes and plays important roles in antitumor immunotherapy by shaping systemic immune responses [6, 7]. Probiotics such as Lactobacillus spp. and Bifidobacterium spp. regarded as live microorganisms are beneficial for human health when given in sufficient quantities [8]. Recent studies have shown that probiotics enhanced host immunity and attenuated tumor growth via tumor immunomodulation [9]. For example, oral administration of Bifidobacterium spp. effectively inhibited tumor growth of a melanoma mouse model by accumulating CD8 $+T$ cells with promotion of antitumor immunity and antiPD-L1 efficacy [6]. Bifidobacterium spp. can also activate natural killer (NK) cells and improve the defense against influenza virus in a murine model [10]. Supplementation of Lactobacillus casei has been reported to enhance anti-tumor immunity in colon cancer model by promoting potent Th1 immune responses, activity of CD8 + T and NK cells infiltration in the tumor tissue [11]. Moreover, several cohort studies indicated that probiotics such as Bifidobacterium and Lactobacillus produced from longterm dietary fiber and yogurt contributed to cancer prevention and treatment $[12,13]$.

Microbiome-derived metabolites such as short chain fatty acids (SCFAs) are concurrently being recognized to be involved in mediating tumor pathogenesis and immunotherapy [14]. Faecalibaculum rodentiuma producing SCFAs has been identified as a novel anti-tumor bacterial strain mainly by inhibiting calcineurin and NAFTc3 activation, controlling protein acetylation and tumor cell proliferation [15]. Previous studies have shown that three butyrate-producing bacterial species were more abundant in better-responded melanoma patients who were treated with anti-CTLA-4 (ipilimumab) [16]. Butyrate was also reported to protect intestinal mucosa against inflammation and carcinogenesis by activating GPCRs (Gpr109a) and inhibiting inflammatory responses of macrophages and dendritic cells in the colon [17]. 
Tryptophan-derived indole derivatives from gut microbiota are key endogenous ligands with a high affinity of aryl hydrocarbon receptor (AhR), which is closely associated with multiple metabolic syndromes such as obesity, cancer, diabetes and high blood pressure $[18,19]$. A variety of intestinal microbes, especially Lactobacillus spp. and Bifidobacterium spp., can produce tryptophan-derived metabolites, such as indole-3-aldehyde (IAId), indole-3-propionic acid (IPA) and indoleacrylic acid (IA) [19-21], which were demonstrated to promote intestinal mucosal barrier integrity and inhibit inflammatory response by stimulating the expression of IL-22 and activating AhR [22]. Of particular note that Lactobacillus reuteri produces indole derivatives of tryptophan such as indole-3-lactic acid, which activate AhR and induce CD $4+\mathrm{CD} 8 \mathrm{aa}+\mathrm{T}$ cells for regulation of intestinal mucosal functions in immunotherapy of inflammatory bowel diseases (IBD) [23]. Inulin naturally sourced from chicory root fiber can produce prebiotics to inhibit melanoma growth by modulating the composition and diversity of gut microbiota and enriching CD4+, CD8 + T cells and plasmacytoid DCs in mouse models [24]. Sporoderm-breaking spores of G. lucidum was also found to significantly modulate gut microbiota structure and composition in tumor-bearing mice and thus increasing the population of CD8 $+T$ cells [25].

Icariin and its derivatives including icaritin, icariside I and icariside II isolated from Herbal Epimedium are natural plant flavonoids with a variety of biological activities such as anti-osteoporosis and anti-tumor effects and commonly used as Chinese traditional medicine [26, 27]. In structure as shown in Fig. 1, icariin (A) is a prenylated flavonol glycoside with rhamnosyl, glucosyl, and methoxy groups. Deglycosylation of icariin will result in icaritin (B). Icariside I (C) and icariside II (D) can be formed when the rhamnose and glucose residues are removed, respectively. Previous studies have mainly focused on the activities of icariin, icaritin and icariside II and showed that these compounds exert their anticancer action through a number of cellular targets and pathways including apoptosis, cell-cycle modulation and immunomodulation [28]. For example, icaritin can effectively inhibit hepatocellular carcinoma (HCC) initiation and malignant growth through IL-6/JAK2/STAT3 pathway signaling [29]. Furthermore, icaritin can also exhibit anti-tumor immunity activity with increasing CD $8+T$ cells infiltration and reducing frequency of MDSCs [30]. Icariside II induced apoptosis in human PC-3 prostate cancer cells by initiating the inhibition of cyclooxygenase-2/prostaglandin E2 (COX-2/PGE2) pathway [31]. Less work, however, have been done on activities of icariside I probably owing to its too low content in Epimedium.

In this study, immunological anti-tumor activity of icariside I was investigated for the first time in a B16F10 melanoma mouse model. Gut microbiota structure and composition in cecal content of mice was firstly analyzed by $16 \mathrm{~S}$ rRNA gene sequence with the goal of screening those bacteria highly associated with tumor immunotherapy. Subsequently, targeted metabolomics approach was employed to quantitatively determine microbiota-derived metabolites such as SCFAs and indole derivatives. The population of multiple lymphocyte subsets in peripheral blood including CD $4+$ and CD8 + T cells, NK and NKT cells were measured with flowcytometry. In addition, histopathological assessments, immunofluorescence quantification and biological assays were also used to verify the inhibitory effects of icariside I on the malignant B16F10 growth of melanoma. These findings demonstrated that icariside I has strong immunological anti-tumor activity through regulation of gut microbiome and host immunity. 


\section{Material And Methods}

\section{Reagents}

Icariside I was obtained from icariin with rhamnosidase hydrolysis as follows: $2 \mathrm{~g}$ of icariin (98\% content) was dissolved in phosphate buffer solution $(\mathrm{pH}=6.8)$ and uniformly mixed with $1 \mathrm{~g}$ of immobilized rhaminoglycoside enzyme following vertex for 24 hours at $60^{\circ} \mathrm{C}$. After the crystals precipitated, the solid powders are filtered and collected, and finally recrystallized with methanol. A total of $1.3 \mathrm{~g}$ of icariin I (95\% content) with a yield of $65 \%$ was obtained from icariin according to specific reaction formula (Fig. S1).

Sodium chloride, Heparin sodium, diethyl ether, isoflurane anesthesia, hydrochloric acid $(\mathrm{HCl})$, $\mathrm{NaH}_{2} \mathrm{PO}_{4} \cdot 2 \mathrm{H}_{2} \mathrm{O}$, and $\mathrm{K}_{2} \mathrm{HPO}_{4} \cdot 3 \mathrm{H}_{2} \mathrm{O}$ were purchased from Sinopharm Chemical Co. Ltd. (Shanghai, China). HPLC-grade water, formic acid (FA), acetonitrile (MeCN), methanol (MeOH) and standards of tryptophan metabolites and short chain fatty acids were obtained from Sigma-Aldrich (Shanghai, China).

\section{Melanoma cells culture}

Murine melanoma B16F10-cell line was obtained from the cell bank of Chinese Academy of Sciences (Shanghai, China). B16F10 cells were cultured in RPMI 1640 medium (Gibco, USA) with 10\% fetal bovine serum (Gibco, USA) and $1 \%$ penicillin/streptomycin (Gibco, USA) at $37^{\circ} \mathrm{C}$ under an atmosphere of $5 \% \mathrm{CO}_{2}$. B16F10 cells were collected by dissociating the cells in trypsin and washed and resuspended in PBS for tumor inoculation.

\section{B16F10 melanoma-bearing mouse model}

The animal experiment was performed according to Chinese National Guidelines and approved by the animal ethics committee of Wuhan Institute of Physics and Mathematics (WIPM, China). A total of 50 female C57BL/6 mice (18-22 g, aged 6-7 weeks) were purchased from Charles River Co. Ltd (Beijing, China) and housed together in groups of three or four mice per cage with a $12 \mathrm{~h}$ light/dark cycle and constant temperature $\left(22 \pm 1^{\circ} \mathrm{C}\right)$ and humidity $(50 \pm 10 \%)$ at Wuhan Institute of Virology (Hubei, China). Mice were allowed to have free access to food and water.

After acclimation for one week, 40 mice were implanted with B16F10 tumor cells by subcutaneous injection at the right flank $\left(0.1 \mathrm{~mL} /\right.$ mouse, $1.0 \times 10^{6}$ cells/mouse $)$ to generate melanoma animal models. The remaining 10 mice were used as normal group. After tumors reached about $50 \mathrm{~mm}^{3}$, the B16F10 tumor-bearing mice were randomly divided into four groups $(n=10)$ as follows: tumor group, low dose (5 $\mathrm{mg} / \mathrm{kg}$ body weight), medium dose ( $20 \mathrm{mg} / \mathrm{kg}$ body weight) and high dose of icariside I ( $80 \mathrm{mg} / \mathrm{kg}$ body weight) treated groups by gavage daily for seven days with corn oil. Meanwhile, normal mice were injected $0.1 \mathrm{~mL}$ of PBS at the similar site and received the same volume of corn oil by gavage. During 7day continuous treatment period, tumor growth was monitored every day with an electronic vernier caliper 
and tumor volume was calculated as $V=0.5 \times a \times b^{2}$, where $a$ and $b$ denote the longer and shorter diameter, respectively.

Mice were sacrificed by cervical dislocation after $8 \mathrm{~h}$ fasting when the tumor of tumor group reached about $1500 \mathrm{~mm}^{3}$. Fecal samples were collected before sacrifice. The tumor tissue was excised, weighed and photographed and part of the tumor tissue was fixed in $10 \%$ formalin solution for histopathological assessment. Other major organs, including heart, liver, lung and kidney, were also collected for histopathological assessment. Peripheral blood was harvested for further analysis. All the samples including plasma, colon, ileum, cecal contents and tumor tissue were stored at $-80{ }^{\circ} \mathrm{C}$ for later experiments.

\section{Histopathological assessment}

Formalin-fixed tumor biopsies were embedded in paraffin wax, sectioned (3-4 $\mu \mathrm{m})$, and stained with H\&Estaining and anti-Ki-67 staining. Tissues from heart, liver, spleen, lung and kidney were also embedded in paraffın wax and stained with hematoxylin and eosin (H\&E). Colonic tissue was fixed in 10\% formalin solution for Periodic acid-Schiff (PAS) staining. All stained sections were observed and photographed under a light microscope (with 200x magnification). Histopathological and immunofluorescence analyses were conducted by a qualified pathologist from Wuhan servicebio technology CO., LTD as a paid service.

\section{Gut microbiota analysis}

For 16S rRNA gene sequencing analysis, total DNA of cecal contents ( 100 mg) was extracted, 16S rRNA gene amplicon sequence library was prepared as described in the protocol of $16 \mathrm{~S}$ Metagenomic Sequencing Library Preparation (Illumina, United States). Paired-end sequencing ( $2 \times 300 \mathrm{bp})$ was performed using an Illumina MiSeq platform by Shanghai Majorbio Bio-pharm Technology Co., Ltd. The preparation of $16 \mathrm{~S}$ rRNA gene amplicon sequence library, statistical analysis and data manipulation were described in the Supporting Information.

\section{Quantification of short chain fatty acids}

Targeted analyses of short chain fatty acids (SCFAs) in the feces of mice were performed on a Shimadzu 2010 Plus GC-MS spectrometer (Shimadzu Scientific Instruments) equipped with a flame ionization detector (FID) and a CP-FFAP CB capillary GC column ( $25 \mathrm{~m} \times 0.32 \mathrm{~mm}, 0.3 \mu \mathrm{m}$, Agilent Technology). The procedure of sample preparation and SCFAs measurements was described previously [32] and in Supplementary Materials.

\section{Quantification of indole metabolites}

Quantification of indole metabolites in fecal, plasma and colon tissues was performed by multiple reaction monitoring (MRM) using an ultrahigh performance liquid chromatography (Agilent 1290) coupled with a 6460 triple quadrupole mass spectrometry (UHPLC-QQQ-MS, Agilent Technologies, Inc.). 
The procedure of sample preparation and indole metabolites measurements are previously described [33] with some improvements and in Supplementary Materials.

\section{Quantification of peripheral blood lymphocyte by flowcytometry}

Peripheral blood was collected from the orbital vein plexus with EDTA-Li micro-anticoagulant tube. Blood sample $(100 \mu \mathrm{L})$ was stained with BV421 anti-mouse CD3e (BD Horizon, NY, USA), FITC anti-mouse CD49b (BD Pharmingen, NY, USA), PE anti-mouse CD4 (BD Pharmingen, NY, USA), PE-Cy7 anti-mouse CD8a (BD Pharmingen, NY, USA) at $4^{\circ} \mathrm{C}$ in dark for 30 min, and then erythrocytes were lysed in Lysing Buffer (BD Pharm Lyse, NY, USA) for 5 min. After washing with pre-cold PBS for two times, $T$ cell subsets in the peripheral blood were accounted with a FACS Canto II cytometer (BD, NY, USA), and the quantitative data were analyzed by FlowJo software (version 7.6.1).

\section{ELISA analysis}

The concentrations of lipopolysaccharide (LPS), CD14, proinflammatory cytokines including IL-1 $\beta$ and IL6 in plasma were measured using ELISA kits (Shanghai Huyu biotechnology Co., Ltd) according to the manufacturer's instructions.

\section{Quantitative real-time PCR (QPCR) and western blot analyses}

The experimental procedure of samples preparation and data analyses was described in Supplementary Materials.

\section{Statistical data analysis}

All experimental values are shown as mean \pm standard deviation. Statistical data analyses and graphical illustrations were performed using GraphPad Prism software (version 7.0). All data between different groups were statistically analyzed using double-tailed Student's t test or Mann-Whitney test. $P$-values < 0.05 were considered as significance.

\section{Results}

\section{Icariside I dose-dependently inhibits tumor growth}

Tumor bearing mice treated with different doses of icariside I $(5 \mathrm{mg} / \mathrm{kg}, 20 \mathrm{mg} / \mathrm{kg}$ and $80 \mathrm{mg} / \mathrm{kg}$ body weight) by gavaging exhibited significant inhibition of tumor growth showing as tumor volume, weight and representative images for tumor (Fig. 2A-C). Specifically, relatively medium dose ( $20 \mathrm{mg} / \mathrm{kg}$ body weight) of icariside I treatment induced an average inhibition rate of $51.72 \%$ of tumor, which is higher than that of $31.03 \%$ and $37.93 \%$ induced by low and high dose of icariside I, respectively (Fig. 2A-B). Icariside I treatments significantly caused pathological changes including cell shrinkage, nuclear condensation and necrosis in tumor cells of tumor tissue in B16F10 bearing mice (Fig. 2D). Icariside I administration also inhibited tumor cell proliferation observed by anti-ki67 staining (Fig. 2D). In addition, 
safety assessments of icariside I in vivo with histopathological examination and body or tissue weight monitoring showed that icariside I is nontoxic or low toxicity to mice within the scope of doses used here by gavaging (Fig. S2).

\section{Icariside I modulates gut microbiota with elevation of probiotics abundance}

High-quality gut microbiota sequences were obtained from all the cecal contents samples of mice by $16 \mathrm{~S}$ rRNA gene sequencing analysis. Microbial a-diversity analysis showed that tumor bearing mice exhibited higher OTU a-diversity indices than that of normal group. Nevertheless, icariside I treatment significantly recovered the unique OTU a-diversity indices in dose-dependent manner (Fig. 3A). The principal coordinates analysis (PCOA) based on Bray-Curtis distance indicated that an obvious shift of gut microbiota community structure occurred between tumor group and normal group $\left(\mathrm{R}^{2}=0.135, P=0.089\right)$; whereas icariside I treatments restored the altered gut microbiota-induced by tumor growth (Fig. 3B, S2AB). Within the intestinal microbiota, Firmicutes, Bacteroidetes, Actinobacteria, Proteobacteria and Tenericutes were observed as the dominant bacteria at the phylum level (Fig. 3C). Of particular note that icariside I treatments significantly recovered the abundance of Actinobacteria and Firmicutes, which were relatively reduced by tumor xenograft (Fig. 3E-F). At genus level, hierarchical clustering analysis represented by heatmap demonstrated that normal and icariside I treatments groups exhibited gut microbiota community shift in contrast to those of tumor group (Fig. 3D). In detail, the most striking changes of genera were typical probiotics such as Lactobacillus and Bifidobacterium, which were significantly higher in both normal and icariside I treatments groups than those in tumor group (Fig. 3G$\mathrm{H})$. Icariside I treatments also induced higher levels of Firmicutes/Bacteroidetes, Adlercreutzia, Allobaculum, Turicibacter and Clostridium and lower level of Bacteroidetes,Ruminococcus and Bacteroides in cecal contents of mice than those in tumor groups (Fig. S3C-J). In addition, the two typical probiotics showed a negative correlation with tumor weight, especially Lactobacillus (Fig. 3I, S3K).

\section{Icariside I improves intestinal short chain fatty acid metabolism}

Short-chain fatty acids (SCFAs), the most abundant microbial metabolites in the intestine, plays an important role in regulation of host immune system by activating $\mathrm{G}$ protein-coupled receptors such as Gpr41 and Gpr43 on intestinal epithelial cells. Here targeted metabolomics analyses showed that icariside I dose-dependently improved the abundance of short-chain fatty acids (SCFAs) including acetate, propionate and butyrate as well as total SCFAs in the feces of mice, which were significantly down-regulated by tumor growth in tumor-bearing mice compared with normal mice (Fig. 4A-B). Spearman's rank correlation analysis indicated that SCFAs positively correlated to probiotics (Lactobacillus and Bifidobacterium) (Fig. 4C-E), which was previously reported to produce SCFAs in the feces of mice [34]. Meanwhile, icariside I administration significantly improved the mRNA levels of Gpr41 and Gpr43 in the colon, further confirmed significant elevation of SCFAs produced from bacterial fermentation in intestine (Fig. 4F-G).

\section{Icariside limproves microbiota-derived indole metabolism and intestinal AhR}


Targeted analysis of microbiota-derived indole metabolites involved in tryptophan metabolism showed that tumor growth obviously impaired indole metabolic pathway (Fig. 5A-D), mainly manifested by significant down-regulation of indole metabolites including indole acetic acid (IAA), indole-3-propionic acid (IPA) and indole in the colon and feces of tumor-bearing mice, which are commonly recognized as endogenous AhR ligands [19, 35]. Icariside I administration markedly improved the levels of these indole metabolites in the colon and feces of tumor-bearing mice (Fig. 5A-D), accompanied with the recovery of the abundance of indole metabolites in plasma (Fig. S4A-B). Consistently, both mRNA and protein levels of AhR were restored in the colon of tumor-bearing mice after icariside I treatment (Fig. 5E), as well as the mRNA level of colonic IL-22 (Fig. S4D), which has been reported to be associated with intestinal homeostasis [22]. Further Spearman's rank correlation analyses showed that both Lactobacillus and Bifidobacterium were positively correlated with multiple microbial-derived indole metabolites (Fig. S4C) and AhR agonists (Fig. 5F-G) in feces of mice. On the contrary, tumor weight negatively correlated with microbiota-derived AhR ligands in feces, colon and plasma of tumor-bearing mice (Fig. S4E-G).

\section{Icariside I improves gut permeability, systemic inflammation and immunity}

Periodic acid-Schiff (PAS) staining of colon showed that tumor-bearing mice exhibited lower number of mucin-producing goblet cells than normal animals (Fig. 6A-B). Consequently, gut permeability was altered due to tumor growth, manifested by significant up-regulation of mRNA levels of markers of gut permeability such as Myosin and Ptprh in the ileum and plasma CD14 of tumor-bearing mice, leading to disruption of intestinal barrier integrity and gut leakiness [19](Fig. 6C-E). Tumor-bearing mice also exhibited high level of bacteria endotoxin LPS in plasma and proinflammatory cytokines including II-6 and $I /-1 \beta$ (Fig. 6F-J) in plasma and tumor tissue, indicating that tumor growth induced systemic inflammation. Icariside I treatments significantly enhanced the amount of goblet cells and ameliorated gut permeability and systemic inflammation of tumor-bearing mice as showing significant improvements of those corresponding markers including ileum Myosin and Ptprh, plasma CD14 and LPS, and proinflammatory cytokines (Fig. 6B-J, S5A-B).

Given close relationship between gut microbiota and derived metabolites and host immunity, we next analyzed the impact of icariside I administration on systemic immunity including multiple lymphocyte subsets by flowcytometry. The results showed that icariside I treatments markedly enhanced the percentage of $C D 4+T$ cell and $C D 8+T$ cell in peripheral blood of tumor-bearing mice, who exhibited lower levels of CD4+ T cell and CD8+ T cell than normal mice (Fig. 7B, E-F). Furthermore, flowcytometry analysis showed that icariside I treatments also enhanced the percentage of natural killer (NK) cells and natural killer T (NKT) cells in peripheral blood of tumor-bearing mice (Fig. 7A, C-D), suggesting that icariside I has noteworthy immunological anti-tumor activity by regulating multiple lymphocyte subsets in the circulatory system.

\section{Discussion}


Epimedium known as Yin Yang Huo or Horny Goat Weed is widely used as a traditional herbal formula in many Asian countries such as China, Japan, and Korea [36]. The main bioactive compounds isolated from Herbal Epimedii include icariin, icaritin, icariside I and icariside II with similar molecular structure. Recently, a large number of studies have focused on the anti-cancer activity of three compounds including icariin [37], icaritin [38], and icariside II [39] in a variety of cancers, such as hepatocellular cancer [29], prostatic cancer [38], and multiple myeloma [40]. Less work, however, have been done on the antitumor activity of icariside I probably due to its too low content in Epimedium. In this study, the underlying mechanisms and the role of the gut microbiota in the immunological anti-tumor activity of icariside I in a B16F10 melanoma model were investigated for the first time using a combination of 16S rRNA gene sequencing, targeted metabolomics and biological assays.

Emerging evidences suggest that the gut microbiota plays an important role in cancer immunotherapy by modulating host immune system [6, 7]. Here icariside I treatment significantly improved tumor xenograftinduced microbiota diversity and composition alterations including Firmicutes, Actinobacteria and Bacteroidetes at phyla levels. It is particularly noteworthy that oral administration of icariside I markedly increased the abundance of Bifidobacterium and Lactobacillus, typical probiotics beneficial for human healthy with immune enhancement, in the cecal content of tumor-bearing mice. Consequently, gut permeability, intestinal barrier integrity, systemic endotoxin LPS and inflammation induced by tumorgrowth were also improved after icariside I treatment. Significant inhibition of tumor growth as showing decreased tumor weight and volume as well as histopathological improvement were expectedly observed. In agreement with our findings, previous investigations found that oral administration of Bifidobacterium alone effectively improved tumor growth control to the same degree as PD-L1 antibody immunotherapy [6]. Oral Lactobacillus can inhibit tumor growth by promoting antitumor immunity and enhancing activity of multiple lymphocyte subsets [41]. Previous studies also revealed that both Bifidobacterium and Lactobacillus have ability to maintain integrity of colonic mucus layer and intestinal permeability for antiinflammation of mice $[42,43]$.

In addition to gut microbiota itself, its derived metabolites such as SCFAs and indole derivatives have also been shown to have immunoregulation function through metabolic signaling pathway. Our results revealed that the levels of SCFAs and indole derivatives in the gut of tumor-bearing mice were significantly recovered after icariside I treatment. Many studies have shown that SCFAs especially butyrate are able to protect the intestinal mucosa from inflammation, improve host immune responses and reduce liver metastasis of colon cancer cells through metabolic signaling regulation $[17,44]$. Here significant elevation of SCFAs indicated activation of intestinal bacterial fermentation process by icariside I, which further confirmed with up-regulation of colonic Gpr41 and Gpr43 mRNAs. Previous studies also showed that microbiota-derived SCFAs can enhance cellular metabolism and memory potential of antigen-activated CD $+\mathrm{T}$ cells in participating in the immune response against intracellular pathogens and tumors [45], as well as promoting activity and differentiation of CD4 + T cells [46]. Most of microbiota-derived indole derivatives are endogenous ligands of AhR, which is involved in host immunometabolism and multiple pathophysiological processes of metabolic diseases such as obesity and cancer [47]. Significant down-regulation of AhR activity with lower levels of gut microbiota-derived 
AhR agonists such as indole derivatives were previously observed in metabolic syndromes such as obesity, diabetes and blood high pressure (BHP) of humans and animal models [19]. Furthermore, AhR activation by tryptophan derivatives (e.g. 6-formylindolo[3,2-b]carbazole) has been reported to enhance NK cells control on tumor growth and maintain NK cells in the liver of mice [48, 49]. In this study, icariside I markedly up-regulated the levels of indole derivatives in fecal and colonic samples of tumor-bearing mice. Concomitantly, colonic AhR expression at both mRNAs and protein levels was also improved after icariside I treatments in a dose-dependent manner. Interestingly, our results also revealed a significant positive correlation between microbiota-derived SCFAs and indole derivatives and Lactobacillus and Bifidobacterium. Actually, numerous studies have reported that microbiota-derived SCFAs and indole derivatives could be produced from probiotics such as Lactobacillus and Bifidobacterium [19, 20, 34].

Immunological anti-tumor activity of icariside I was further directly supported by significant elevation of multiple lymphocytes including CD4 + T, CD 8 + T, NK and NKT cells in peripheral-blood of tumor-bearing mice after icariside I treatment, indicating enhancement of host immune function by icariside I. It is well known that adaptive $T$ cells including $C D 4+T$ and $C D 8+T$ cells can mediate cytotoxic responses for antitumor immunotherapy [50]. Specifically, CD8 $+T$ cells are currently regarded as the most effective elements for tumor destruction, which can be activated by the tumor cell-expressing surface molecules leading to tumor cell apoptosis [51]. Melanoma patients with higher number of CD $8+T$ cells are more likely to gain better outcomes concerning immunotherapy and therefore are often valued as immunotherapeutic targets in tumor cell lysis [52]. NK and NKT cells are able to recognize and spontaneously kill tumor cells without prior sensitization by secreting multiple cytokines and chemokines such as IFNy and CC-chemokine ligand 3 (CCL3) and activating the caspases of tumor cells [53]. In recent years, CD4 + T cells have received increasing attention due to their functional diversity in directly or indirectly regulating anti-tumor immunity, such as promoting the activation and differentiation of CD8 $+T$ cells and enhancing the activity of killing tumor cells $[54,55]$.

Collectively, this study revealed that icariside I as a novel compound isolated from Epimedium significantly improves host intestinal barrier and immunity through regulation of gut microbiota and its derived metabolites. Specifically, icariside I treatment markedly upregulated levels of probiotics such as Lactobacillus and Bifidobacterium and their metabolites including SCFAs and indole derivatives, which participate in host metabolic regulation through colonic Gpr41/Gpr43 and $A h R$ signaling. These findings suggested that icariside I ( 5,20 and $80 \mathrm{mg} / \mathrm{kg}$ body weight) exhibits low toxicity and immunological antitumor activity potently improving host immunity and inhibiting tumor growth. Clinical studies focused on tumor immunomodulatory therapy of icariside I are indeed warranted and should shed some light on how this compound may be developed as a novel therapeutic agent for cancer.

\section{Abbreviations}

SCFAs: short chain fatty acids; NK cell: natural killer cells; NKT cells: natural killer T cells; BRAF: v-raf murine sarcoma viral oncogene homolog B; MEK: mitogen-activated proteinkinase kinase; CTLA-4: cytotoxic T-lymphocyte-associated antigen-4; PD-1:programmed cell-death protein 1; FDA: the US Food 
and Drug Administration; GPCRs: G protein coupling receptors; AhR: aryl hydrocarbon receptor; IBD: inflammatory bowel diseases; DCs: dendritic cells; HCC: hepatocellular carcinoma; MDSCs: Myeloidderived suppressor cells; LPS: lipopolysaccharide; IL-1ß:interleukin-1 a; IL-6: interleukin-6; MRM: multiple reaction monitoring; PCoA: principal coordinates analysis; QPCR: Real-Time Polymerase Chain Reaction; Gpr41:G protein-coupled receptor 41; Gpr43:G protein-coupled receptor 43; ILA:Indole-3-Lactic Acid; IA: Indole Acrylic Acid; IPA:Indole-3-Propionic Acid; IAA: Indole Acetic Acid; IAld:Indole-3-Aldehyde; PAS: Periodic acid-Schiff; Ptprh, protein tyrosine phosphatase, receptor type, $\mathrm{H} ; \mathrm{CCL}$ :CC-chemokine ligand 3; GAPDH:Glyceraldehyde-3-phosphate dehydrogenase.

\section{Declarations}

\section{Acknowledgements}

This work was financially supported by National Key Research and Development Project (2018YFE0110800), Foshan Core Technology Tackling Key Project (1920001000262), China Postdoctoral Science Foundation (2019M662893) and the National Natural Science Foundation of China (21635006).

\section{Competing interests}

The authors declare no competing financial interest.

\section{Author contributors}

Z.L. and C.G. designed the study and wrote the manuscript. C.G.,C.Z., S.Z. and L.H. performed animal husbandry, melanoma mouse model and icariside I treatment experiments. C.G., C.C., Y.P., W.F., L.C., D.M. and S.Y. finished metabolomics experiments, biological assays and analyzed the data. L.Y., and Z.J. participated in discussion and editing. Z.L. and S.Z. directed this project and provided valuable feedback on the manuscript.

\section{References}

1. Brighton HE, Angus SP, Bo T, Roques J, Tagliatela AC, Darr DB, Karagoz K, Sciaky N, Gatza ML, Sharpless NE, et al. New Mechanisms of Resistance to MEK Inhibitors in Melanoma Revealed by Intravital Imaging. Cancer Res. 2018;78:542-57.

2. Azijli K, Stelloo E, Peters GJ. AJM: New developments in the treatment of metastatic melanoma: immune checkpoint inhibitors and targeted therapies. Anticancer Res. 2014;34:1493-505.

3. Luke JJ, Flaherty KT, Ribas A, Long GV. Targeted agents and immunotherapies: optimizing outcomes in melanoma. Nat Rev Clin Oncol. 2017;14:463-82.

4. Callahan MK, Postow MA, Wolchok JD. Targeting T Cell Co-receptors for Cancer Therapy. Immunity. 2016;44:1069-78. 
5. Robert C, Schachter J, Long GV, Arance A, Grob JJ, Mortier L, Daud A, Carlino MS, McNeil C, Lotem M, et al. Pembrolizumab versus Ipilimumab in Advanced Melanoma. N Engl J Med. 2015;372:2521-32.

6. Sivan A, Corrales L, Hubert N, Williams JB, Aquino-Michaels K, Earley ZM, Benyamin FW, Man Lei Y, Jabri B, Alegre $M-L$, et al. Commensal Bifidobacterium promotes antitumor immunity and facilitates anti-PD-L1 efficacy. Science. 2015;350:1084-9.

7. Gopalakrishnan V, Spencer CN, Nezi L, Reuben A, Andrews MC, Karpinets TV, Prieto PA, Vicente D, Hoffman K, Wei SC, et al. Gut microbiome modulates response to anti-PD-1 immunotherapy in melanoma patients. Science. 2018;359:97-103.

8. Hill C, Guarner F, Reid G, Gibson GR, Merenstein DJ, Pot B, Morelli L, Canani RB, Flint HJ, Salminen S, et al. Expert consensus document. The International Scientific Association for Probiotics and Prebiotics consensus statement on the scope and appropriate use of the term probiotic. Nat Rev Gastroenterol Hepatol. 2014;11:506-14.

9. Zhong L, Zhang X, Covasa M. Emerging roles of lactic acid bacteria in protection against colorectal cancer. World J Gastroenterol. 2014;20:7878-86.

10. Kawahara T, Takahashi T, Oishi K, Tanaka H, Masuda M, Takahashi S, Takano M, Kawakami T, Fukushima K, Kanazawa H, Suzuki T. Consecutive oral administration of Bifidobacterium longum MM-2 improves the defense system against influenza virus infection by enhancing natural killer cell activity in a murine model. Microbiol Immunol. 2015;59:1-12.

11. Aindelis G, Tiptiri-Kourpeti A, Lampri E, Spyridopoulou K, Lamprianidou E, Kotsianidis I, Ypsilantis P, Pappa A, Chlichlia K. Immune Responses Raised in an Experimental Colon Carcinoma Model Following Oral Administration of Lactobacillus casei. Cancers (Basel). 2020;12:368.

12. Yang JJ, Yu D, Xiang Y-B, Blot W, White E, Robien K, Sinha R, Park Y, Takata Y, Lazovich D, et al. Association of Dietary Fiber and Yogurt Consumption With Lung Cancer Risk: A Pooled Analysis. JAMA Oncology. 2020;6:e194107-7.

13. Zheng X, Wu K, Song M, Ogino S, Fuchs CS, Chan AT, Giovannucci EL, Cao Y, Zhang X. Yogurt consumption and risk of conventional and serrated precursors of colorectal cancer. Gut. 2020;69:970-2.

14. Gilbert JA, Blaser MJ, Caporaso JG, Jansson JK, Lynch SV, Knight R. Current understanding of the human microbiome. Nat Med. 2018;24:392-400.

15. Zagato E, Pozzi C, Bertocchi A, Schioppa T, Saccheri F, Guglietta S, Fosso B, Melocchi L, Nizzoli G, Troisi $\mathrm{J}$, et al. Endogenous murine microbiota member Faecalibaculum rodentium and its human homologue protect from intestinal tumour growth. Nat Microbiol. 2020;5:511-24.

16. Chaput N, Lepage P, Coutzac C, Soularue E, Le Roux K, Monot C, Boselli L, Routier E, Cassard L, Collins $\mathrm{M}$, et al. Baseline gut microbiota predicts clinical response and colitis in metastatic melanoma patients treated with ipilimumab. Ann Oncol. 2017;28:1368-79.

17. Singh N, Gurav A, Sivaprakasam S, Brady E, Padia R, Shi H, Thangaraju M, Prasad PD, Manicassamy $\mathrm{S}$, Munn DH, et al. Activation of Gpr109a, receptor for niacin and the commensal metabolite butyrate, suppresses colonic inflammation and carcinogenesis. Immunity. 2014;40:128-39. 
18. Metidji A, Omenetti S, Crotta S, Li Y, Nye E, Ross E, Li V, Maradana MR, Schiering C, Stockinger B. The Environmental Sensor AHR Protects from Inflammatory Damage by Maintaining Intestinal Stem Cell Homeostasis and Barrier Integrity. Immunity. 2018;49:353-62 e355.

19. Natividad JM, Agus A, Planchais J, Lamas B, Jarry AC, Martin R, Michel ML, Chong-Nguyen C, Roussel R, Straube M, et al. Impaired Aryl Hydrocarbon Receptor Ligand Production by the Gut Microbiota Is a Key Factor in Metabolic Syndrome. Cell Metab. 2018;28:737-49 e734.

20. Aragozzini F, Ferrari A, Pacini N, Gualandris R. Indole-3-lactic acid as a tryptophan metabolite produced by Bifidobacterium spp. Appl Environ Microbiol. 1979;38:544-6.

21. Russell WR, Duncan SH, Scobbie L, Duncan G, Cantlay L, Calder AG, Anderson SE, Flint HJ. Major phenylpropanoid-derived metabolites in the human gut can arise from microbial fermentation of protein. Mol Nutr Food Res. 2013;57:523-35.

22. Zelante T, lannitti RG, Cunha C, De Luca A, Giovannini G, Pieraccini G, Zecchi R, D'Angelo C, MassiBenedetti C, Fallarino F, et al. Tryptophan catabolites from microbiota engage aryl hydrocarbon receptor and balance mucosal reactivity via interleukin-22. Immunity. 2013;39:372-85.

23. Cervantes-Barragan L, Chai JN, Tianero MD, Di Luccia B, Ahern PP, Merriman J, Cortez VS, Caparon MG, Donia MS, Gilfillan S, et al. Lactobacillus reuteri induces gut intraepithelial CD4 + CD8aa + T cells. Science. 2017;357:806-10.

24. Li Y, Elmen L, Segota I, Xian Y, Tinoco R, Feng Y, Fujita Y, Segura Munoz RR, Schmaltz R, Bradley LM, et al. Prebiotic-Induced Anti-tumor Immunity Attenuates Tumor Growth. Cell Rep. 2020;30:1753-66 e1756.

25. Su J, Su L, Li D, Shuai O, Zhang Y, Liang H, Jiao C, Xu Z, Lai Y, Xie Y. Antitumor Activity of Extract From the Sporoderm-Breaking Spore of Ganoderma lucidum: Restoration on Exhausted Cytotoxic $T$ Cell With Gut Microbiota Remodeling. Front Immunol. 2018;9:1765.

26. Huang X, Zhu D, Lou Y. A novel anticancer agent, icaritin, induced cell growth inhibition, G1 arrest and mitochondrial transmembrane potential drop in human prostate carcinoma PC-3 cells. Eur J Pharmacol. 2007;564:26-36.

27. Zhang DW, Cheng Y, Wang NL, Zhang JC, Yang MS, Yao XS. Effects of total flavonoids and flavonol glycosides from Epimedium koreanum Nakai on the proliferation and differentiation of primary osteoblasts. Phytomedicine. 2008;15:55-61.

28. Tan HL, Chan KG, Pusparajah P, Saokaew S, Duangjai A, Lee LH, Goh BH. Anti-Cancer Properties of the Naturally Occurring Aphrodisiacs: Icariin and Its Derivatives. Front Pharmacol. 2016;7:191.

29. Zhao H, Guo Y, Li S, Han R, Cai J. A novel anti-cancer agent Icaritin suppresses hepatocellular carcinoma initiation and malignant growth through the IL-6/Jak2/Stat3 pathway. Oncotarget. 2015;6:31927-43.

30. Hao H, Zhang Q, Zhu H, Wen Y, Qiu D, Xiong J, Fu X, Wu Y, Meng K, Li J. Icaritin promotes tumor T-cell infiltration and induces antitumor immunity in mice. Eur J Immunol. 2019;49:2235-44.

31. Lee KS, Lee HJ, Ahn KS, Kim SH, Nam D, Kim DK, Choi DY, Ahn KS, Lu J, Kim SH. Cyclooxygenase2/prostaglandin E2 pathway mediates icariside II induced apoptosis in human PC-3 prostate cancer 
cells. Cancer Lett. 2009;280:93-100.

32. Demehri FR, Frykman PK, Cheng Z, Ruan C, Wester T, Nordenskjold A, Kawaguchi A, Hui TT, Granstrom AL, Funari V, et al. Altered fecal short chain fatty acid composition in children with a history of Hirschsprung-associated enterocolitis. J Pediatr Surg. 2016;51:81-6.

33. Fuertig R, Ceci A, Camus SM, Bezard E, Luippold AH, Hengerer B. LC-MS/MS-based quantification of kynurenine metabolites, tryptophan, monoamines and neopterin in plasma, cerebrospinal fluid and brain. Bioanalysis. 2016;8:1903-17.

34. Koh A, De Vadder F, Kovatcheva-Datchary P, Backhed F. From Dietary Fiber to Host Physiology: ShortChain Fatty Acids as Key Bacterial Metabolites. Cell. 2016;165:1332-45.

35. Agus A, Planchais J, Sokol H. Gut Microbiota Regulation of Tryptophan Metabolism in Health and Disease. Cell Host Microbe. 2018;23:716-24.

36. Ma H, He X, Yang Y, Li M, Hao D, Jia Z. The genus Epimedium: an ethnopharmacological and phytochemical review. J Ethnopharmacol. 2011;134:519-41.

37. Di S, Fan C, Yang Y, Jiang S, Liang M, Wu G, Wang B, Xin Z, Hu W, Zhu Y, et al. Activation of endoplasmic reticulum stress is involved in the activity of icariin against human lung adenocarcinoma cells. Apoptosis. 2015;20:1229-41.

38. Sun F, Zhang ZW, Tan EM, Lim ZLR, Li Y, Wang XC, Chua SE, Li J, Cheung E, Yong EL. Icaritin suppresses development of neuroendocrine differentiation of prostate cancer through inhibition of IL-6/STAT3 and Aurora kinase A pathways in TRAMP mice. Carcinogenesis. 2016;37:701-11.

39. Geng YD, Zhang C, Shi YM, Xia YZ, Guo C, Yang L, Kong LY. Icariside II-induced mitochondrion and lysosome mediated apoptosis is counterbalanced by an autophagic salvage response in hepatoblastoma. Cancer Lett. 2015;366:19-31.

40. Zhu S, Wang Z, Li Z, Peng H, Luo Y, Deng M, Li R, Dai C, Xu Y, Liu S. Icaritin suppresses multiple myeloma, by inhibiting IL-6/JAK2/STAT3. Oncotarget. 2015;6:10460-72.

41. Hu J, Wang C, Ye L, Yang W, Huang H, Meng F, Shi S, Ding Z. Anti-tumour immune effect of oral administration of Lactobacillus plantarum to CT26 tumour-bearing mice. J Biosci. 2015;40:269-79.

42. Pan F, Zhang L, Li M, Hu Y, Zeng B, Yuan H, Zhao L, Zhang C. Predominant gut Lactobacillus murinus strain mediates anti-inflammaging effects in calorie-restricted mice. Microbiome. 2018;6:54.

43. Schroeder BO, Birchenough GMH, Stahlman M, Arike L, Johansson MEV, Hansson GC, Backhed F. Bifidobacteria or Fiber Protects against Diet-Induced Microbiota-Mediated Colonic Mucus Deterioration. Cell Host Microbe. 2018;23:27-40 e27.

44. Ma X, Zhou Z, Zhang X, Fan M, Hong Y, Feng Y, Dong Q, Diao H, Wang G. Sodium butyrate modulates gut microbiota and immune response in colorectal cancer liver metastatic mice. Cell Biol Toxicol. 2020.

45. Bachem A, Makhlouf C, Binger KJ, de Souza DP, Tull D, Hochheiser K, Whitney PG, Fernandez-Ruiz D, Dahling S, Kastenmuller W, et al. Microbiota-Derived Short-Chain Fatty Acids Promote the Memory Potential of Antigen-Activated CD8(+) T Cells. Immunity. 2019;51:285-97. e285. 
46. Park J, Goergen CJ, HogenEsch H, Kim CH. Chronically Elevated Levels of Short-Chain Fatty Acids Induce T Cell-Mediated Ureteritis and Hydronephrosis. J Immunol. 2016;196:2388-400.

47. Liu Y, Hou Y, Wang G, Zheng X, Hao H. Gut Microbial Metabolites of Aromatic Amino Acids as Signals in Host-Microbe Interplay. Trends Endocrinol Metab. 2020.

48. Shin JH, Zhang L, Murillo-Sauca O, Kim J, Kohrt HE, Bui JD, Sunwoo JB. Modulation of natural killer cell antitumor activity by the aryl hydrocarbon receptor. Proc Natl Acad Sci U S A. 2013;110:12391-6.

49. Zhang LH, Shin JH, Haggadone MD, Sunwoo JB. The aryl hydrocarbon receptor is required for the maintenance of liver-resident natural killer cells. J Exp Med. 2016;213:2249-57.

50. Matsushita H, Vesely MD, Koboldt DC, Rickert CG, Uppaluri R, Magrini VJ, Arthur CD, White JM, Chen YS, Shea LK, et al. Cancer exome analysis reveals a T-cell-dependent mechanism of cancer immunoediting. Nature. 2012;482:400-4.

51. Mittal D, Gubin MM, Schreiber RD, Smyth MJ. New insights into cancer immunoediting and its three component phases-elimination, equilibrium and escape. Curr Opin Immunol. 2014;27:16-25.

52. Tumeh PC, Harview CL, Yearley JH, Shintaku IP, Taylor EJ, Robert L, Chmielowski B, Spasic M, Henry G, Ciobanu V, et al. PD-1 blockade induces responses by inhibiting adaptive immune resistance. Nature. 2014;515:568-71.

53. Lanier LL. Up on the tightrope: natural killer cell activation and inhibition. Nat Immunol. 2008;9:495502.

54. West NR, Kost SE, Martin SD, Milne K, Deleeuw RJ, Nelson BH, Watson PH. Tumour-infiltrating FOXP3(+) lymphocytes are associated with cytotoxic immune responses and good clinical outcome in oestrogen receptor-negative breast cancer. Br J Cancer. 2013;108:155-62.

55. Oh DY, Kwek SS, Raju SS, Li T, McCarthy E, Chow E, Aran D, llano A, Pai CS, Rancan C, et al. Intratumoral CD4(+) T Cells Mediate Anti-tumor Cytotoxicity in Human Bladder Cancer. Cell. 2020;181:1612-25 e1613.

\section{Figures}


A<smiles>COc1ccc(-c2oc3c(CC=C(C)C)c(OC4O[C@H](CO)[C@@H](O)[C@H](O)[C@H]4O)cc(O)c3c(=O)c2O[C@@H]2O[C@H](C)[C@@H](O)[C@H](O)[C@H]2O)cc1</smiles>

C<smiles>COc1ccc(-c2oc3c(CC=C(C)C)c(OC4O[C@H](CO)[C@@H](O)[C@H](O)[C@H]4O)cc(O)c3c(=O)c2O)cc1</smiles>

Icariside I
B

$\mathrm{HO}$<smiles>COc1ccc(-c2oc3c(CC=C(C)C)c(I)cc(O)c3c(=O)c2O)cc1</smiles>

Icartin

D<smiles>COc1ccc(-c2oc3c(CC=C(C)C)c(O)cc(O)c3c(=O)c2O[C@@H]2O[C@H](C)[C@@H](O)[C@H](O)[C@H]2O)cc1</smiles>

Icariside II

Figure 1

Chemical structures of icariin and its derivatives isolated from Herba Epimedii. (A) Icariin. (B) icaritin. (C) icariside I. (D) icariside II. 

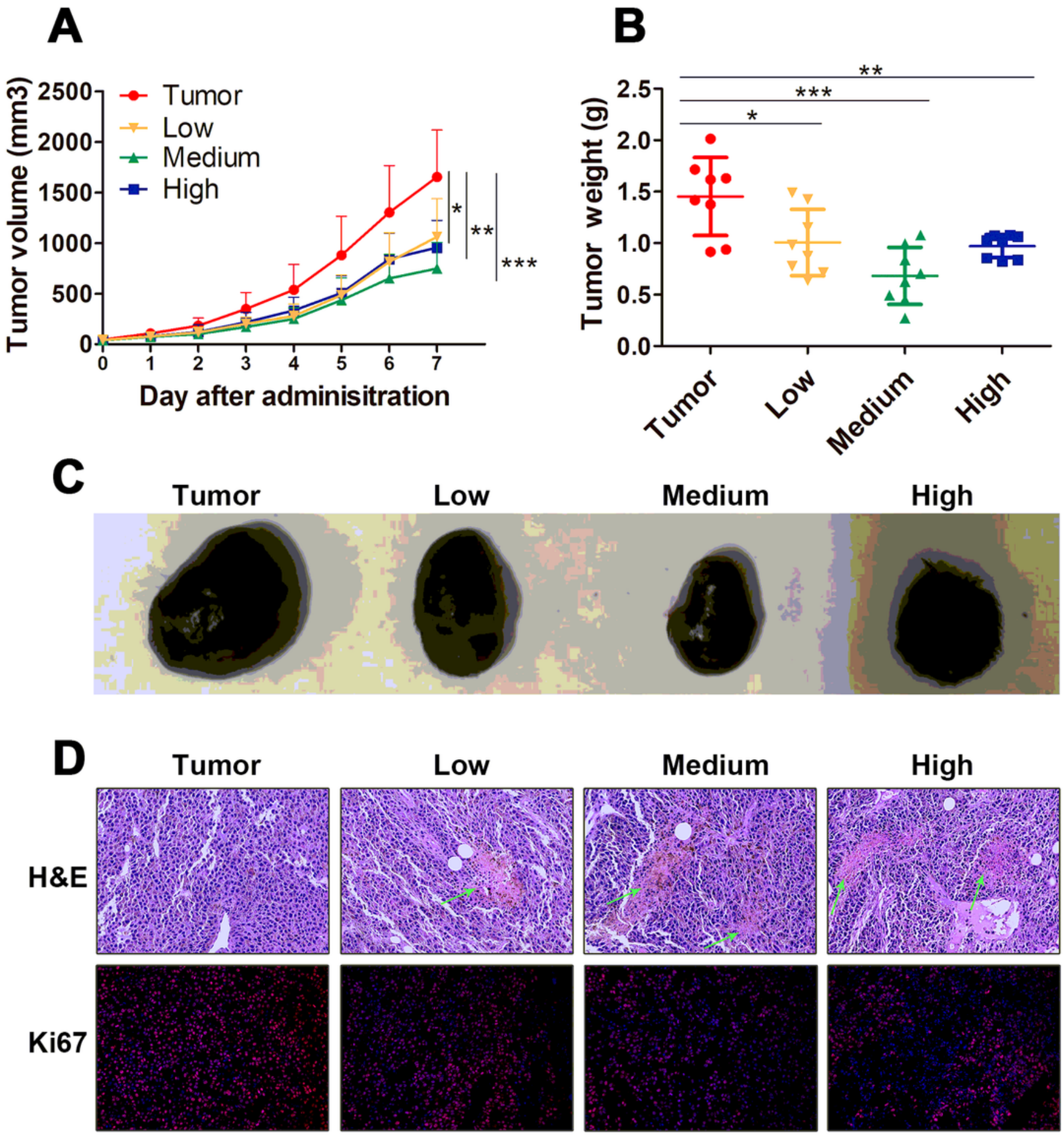

Figure 2

Icariside I inhibits B16F10 melanoma growth in vivo. (A) Tumor volume was measured with a caliper every day for a week. (B) The weight of the tumors was measured. (C) Representative images for tumor from each group. (D) Representative H\&E staining images and immunofluorescence staining images of cell proliferation marker (Ki-67) in tumor tissues from each group (200X). Values were represented the means $\pm S D(n=10) .{ }^{*} p<0.05,{ }^{*} p<0.01$ and ${ }^{* \star *} p<0.001$ versus Tumor group. 

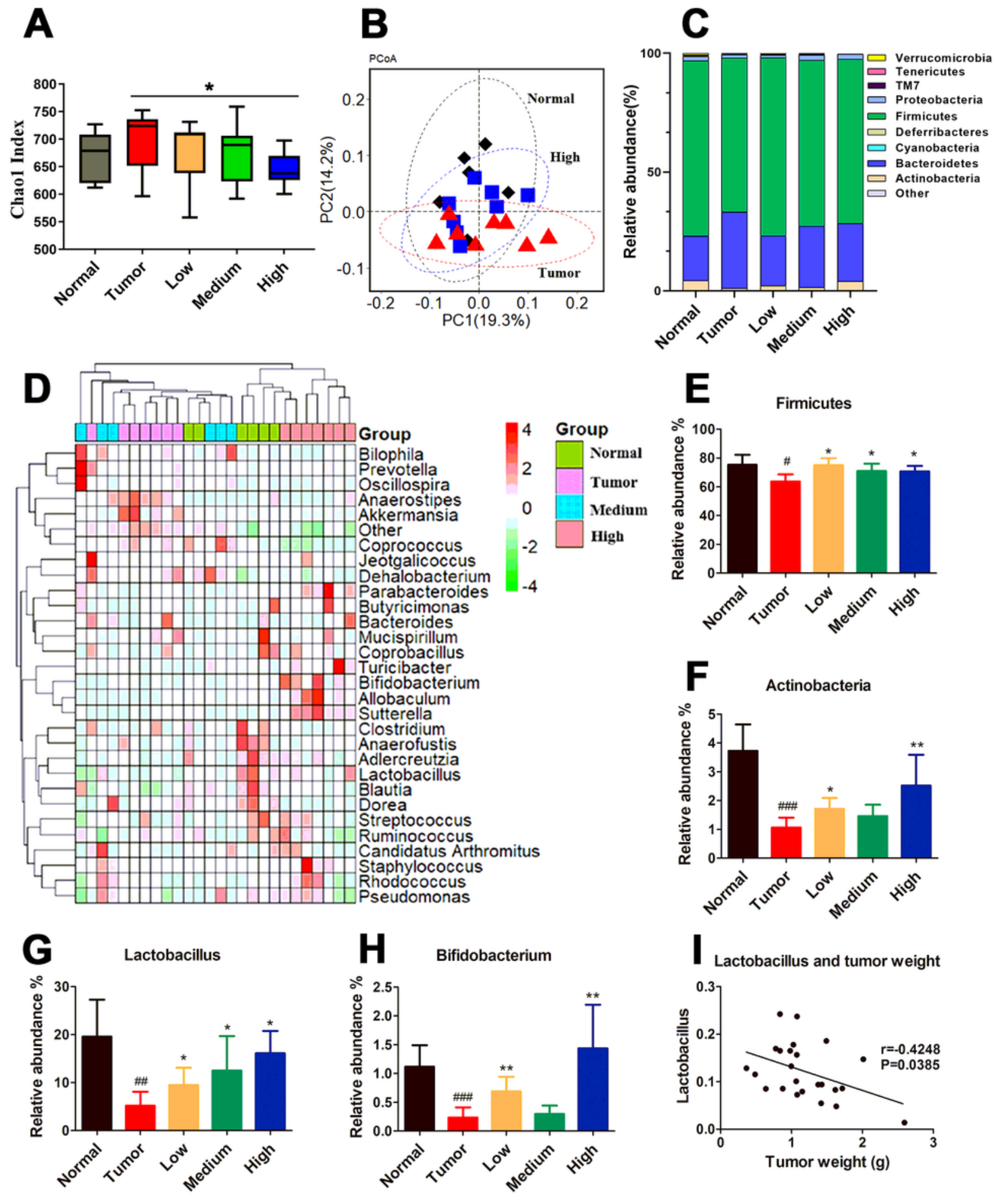

\section{Figure 3}

Icariside I modulates the gut microbiota in the cecal contents of each groups. (A) a-diversity analysis based on observed OTUs. (B) $\beta$-diversity index analysis based on Bray-Curtis dissimilarities for Normal and Tumor groups and the group of High dose of Icariside I. (C) Relative abundance of gut microbiota at phylum level. (D) Heatmap of taxa abundance at genus level for Normal and Tumor groups and the groups of Medium and High dose of Icariside I. (E-F) 16S rRNA gene sequencing analysis of Firmicutes 
and Actinobacteria abundance. (G-H) 16S rRNA gene sequencing analysis of Lactobacillus, Bifidobacterium. (I) Spearman correlation of the abundance of Lactobacillius and tumor weight. Values were represented the means $\pm S D$. \#p $<0.05$, \#\#p $<0.01$ and \#\#\#p $<0.001$ versus normal group; $* p<$ $0.05,{ }^{* *} p<0.01$ and ${ }^{* * *} \mathrm{p}<0.001$ versus tumor group.
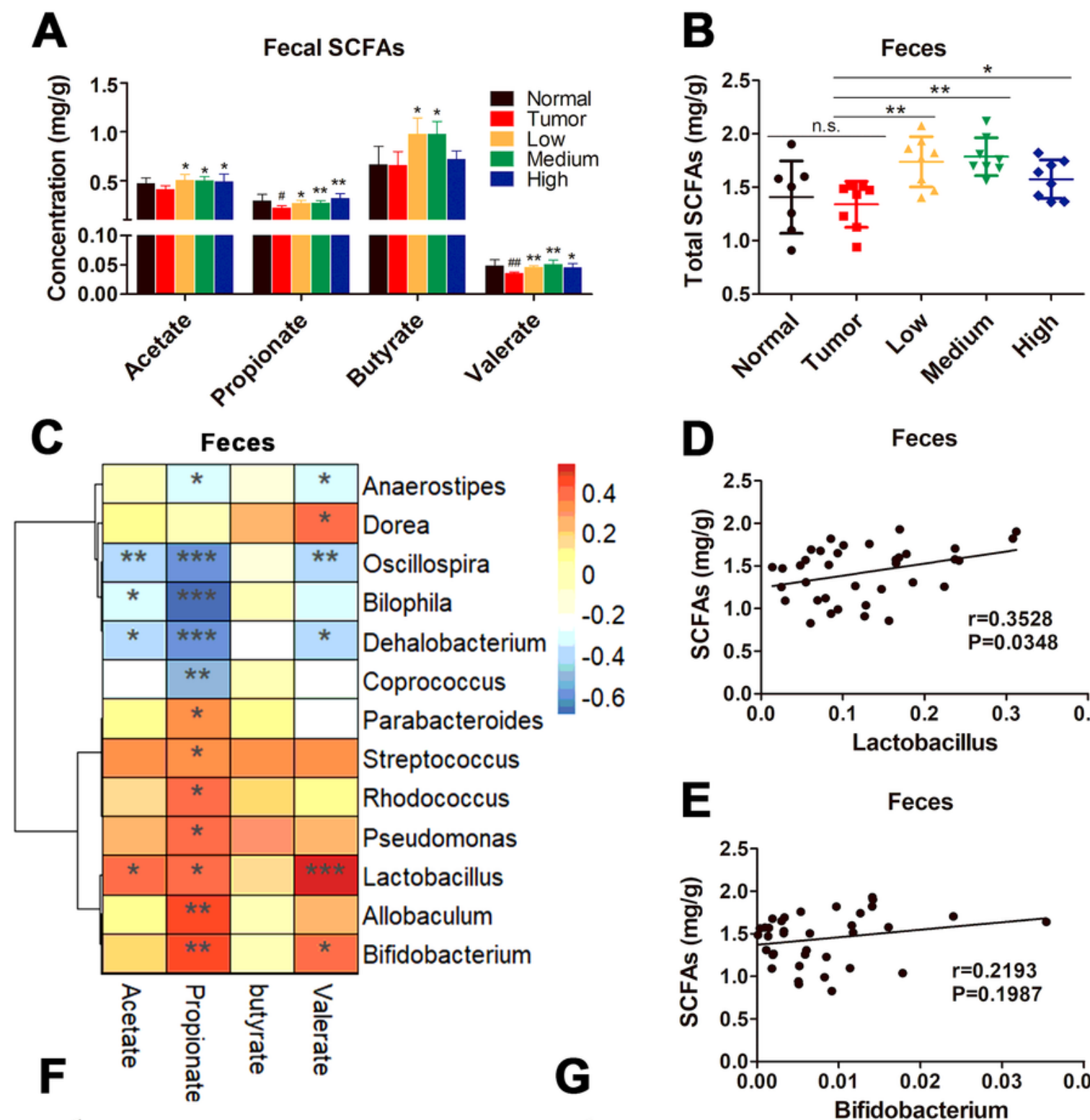

D

Feces
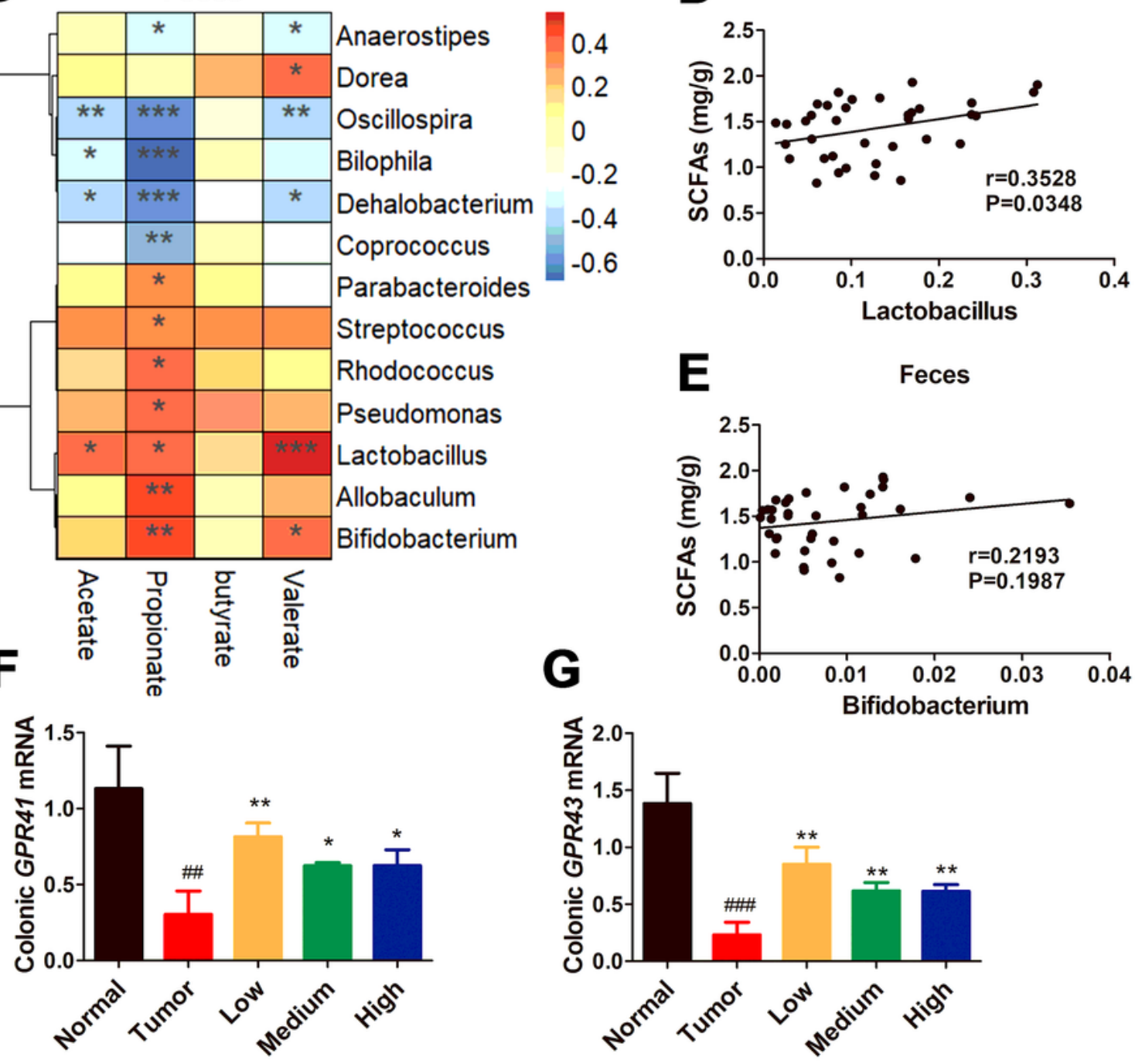

Figure 4 
Icariside I improves intestinal short chain fatty acid metabolism. (A) The concentration of SCFAs (acetate, propionate, butyrate and valerate) from fecal samples of each group. (B) Total concentrations of SCFAs in feces. (C) The correlation heatmap based on Spearman correlation analysis between the gut microbiota and microbiota-derived SCFAs in feces. (D-E) Correlation analysis between the abundance of Lactobacillius or Bifidobacterium and the total SCFAs. (F-G) Colonic mRNA levels of Gpr41 and Gpr43. Values were represented the means $\pm S D(n=10)$. $\# p<0.05$, \#\#p $<0.01$ and \#\#\#p $<0.001$ versus Normal group; ${ }^{*} p<0.05, * * p<0.01$ and $* * * p<0.001$ versus Tumor group.

A
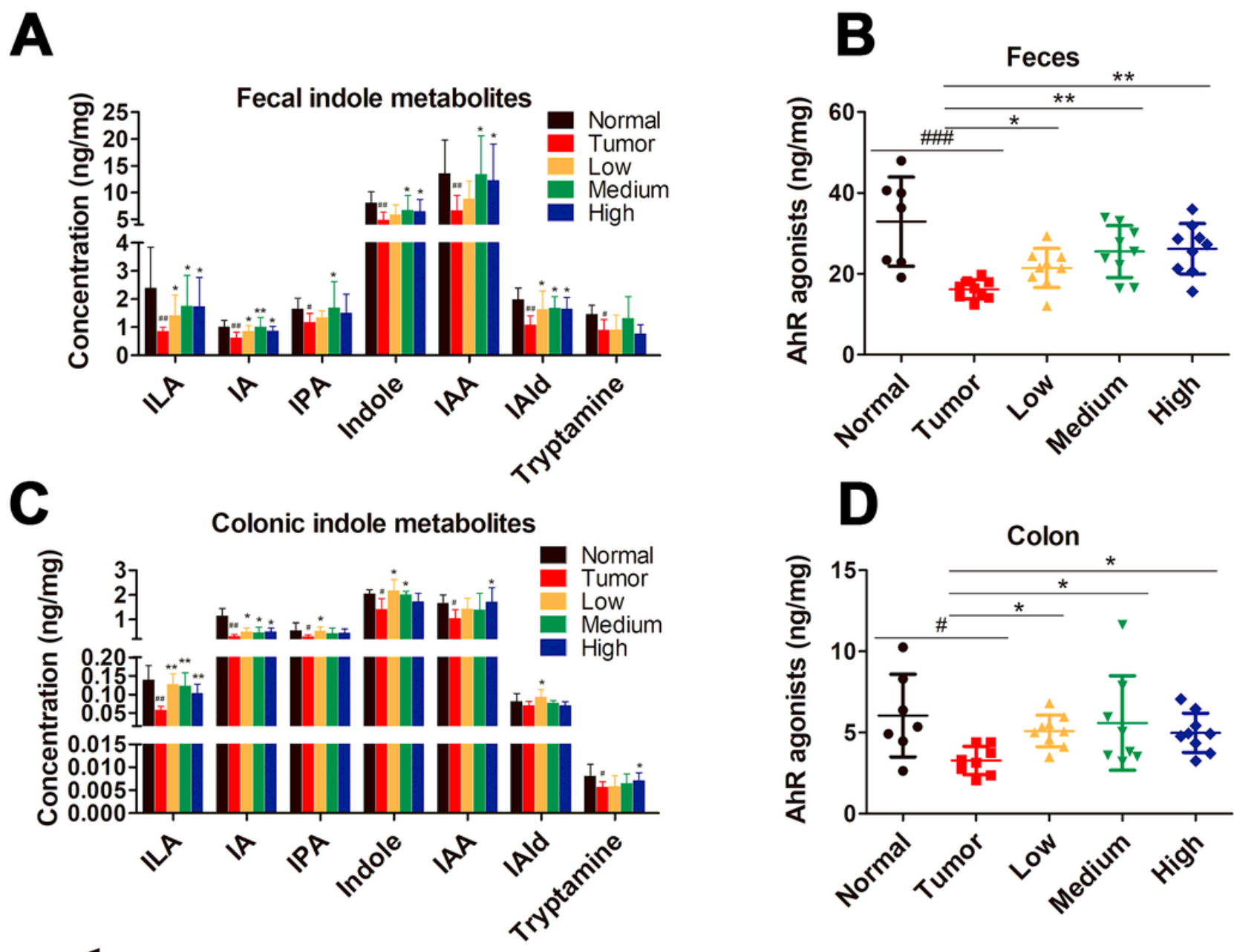

C
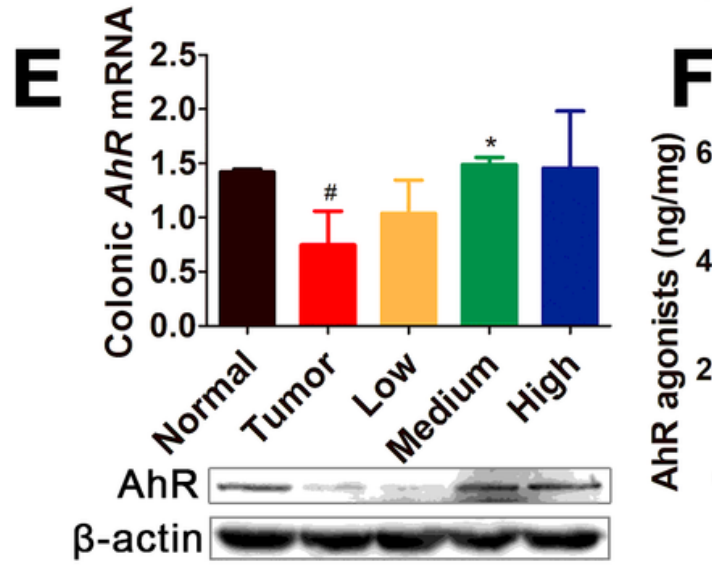

Feces

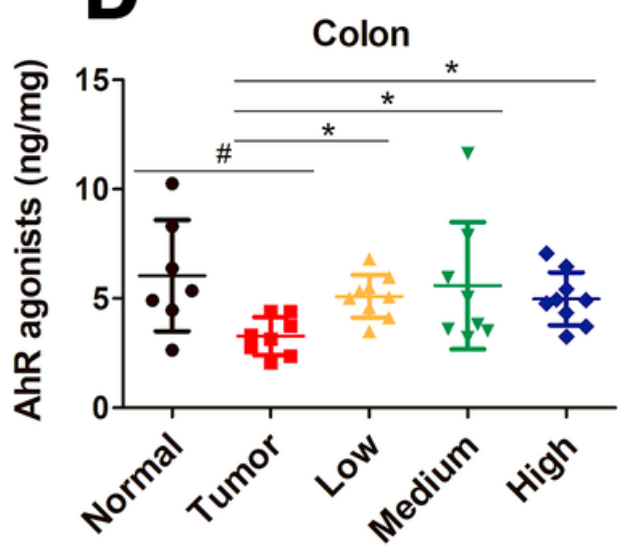

Figure 5 
Icariside I improves microbiota-derived indole metabolism and intestinal AhR activity. (A, C) The concentrations of microbiota-derived indole metabolites (ILA, IA, IPA, Indole, IAA, IAld and Tryptamine) from fecal and colonic samples of each group. (B, D) Total concentrations of AHR agonists (IA, IPA, Indole, IAA and IAId) from fecal and colonic samples. (E) Colonic mRNA and protein levels of AhR. (F-G) Spearman correlation of fecal AhR agonists and the abundance of Lactobacillius or Bifidobacterium. Values were represented the means \pm SD $(n=10)$. \#p $<0.05$, \#\#p $<0.01$ and \#\#\#p $<0.001$ versus Normal group; ${ }^{*}<0.05,{ }^{* *} p<0.01$ and $* * * p<0.001$ versus Tumor group.

A

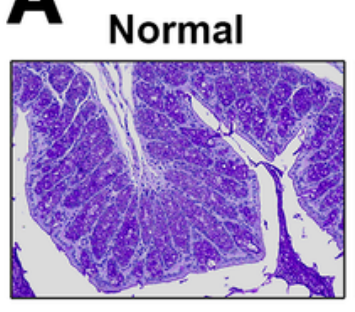

Tumor

Low

Medium
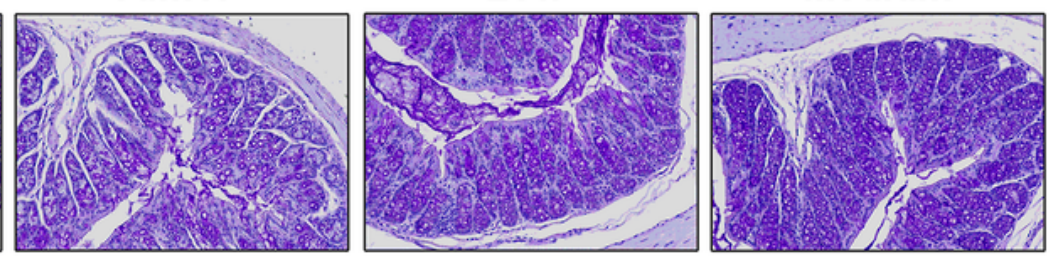

High

B

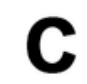

D
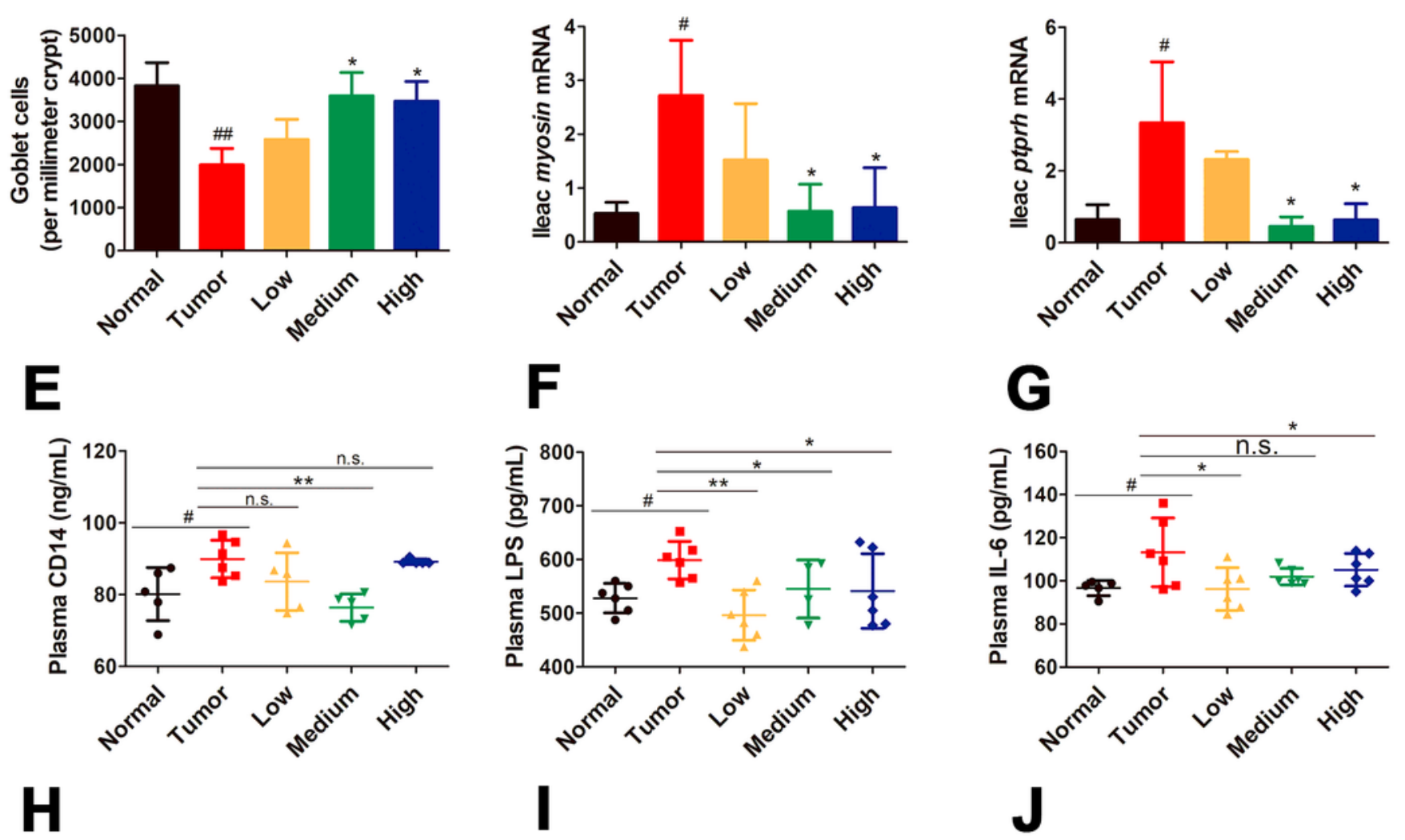

H
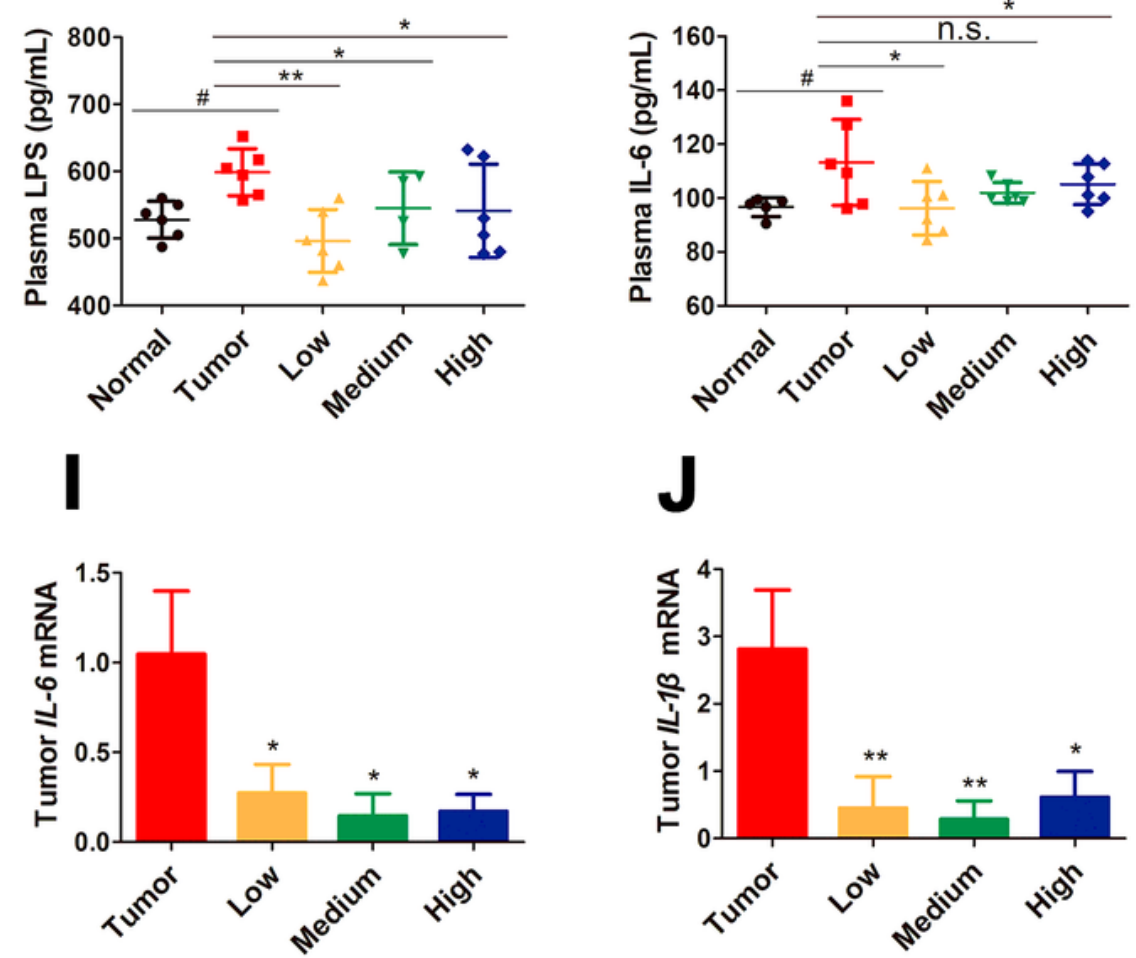


\section{Figure 6}

Icariside I improves gut permeability and systemic inflammation. (A) Representative periodic acid-schiff (PAS) staining images of colons from each group (200X). (B) The number of colonic goblet cells. (C-E) Gut permeability markers including mRNA level of Myosin and Ptprh in the lleum and concentration of soluble CD14 in plasma. (F-H) Systemic inflammation markers including concentration of LPS, IL-6 and IL-1 $\beta$ in plasma. (I-J) The mRNA levels of IL- 6 and IL-1 $\beta$ in tumor tissue. Values were represented the means $\pm S D(n=6)$. $\# p<0.05, \# \# p<0.01$ and $\# \# \# p<0.001$ versus Normal group; ${ }^{*} p<0.05,{ }^{*} p<0.01$ and $* \star * p<0.001$ versus Tumor group. 
A

Normal

Tumor

Low

Medium
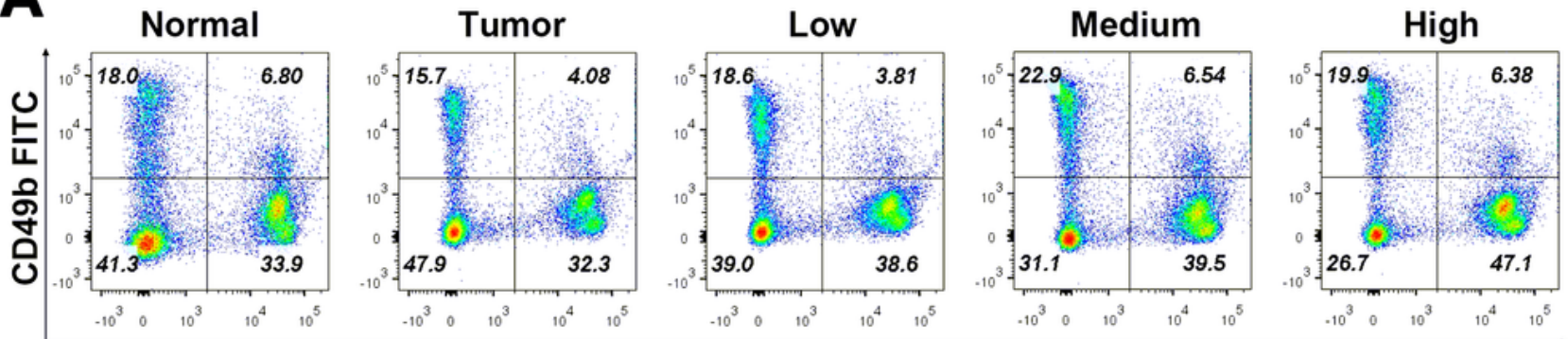

B
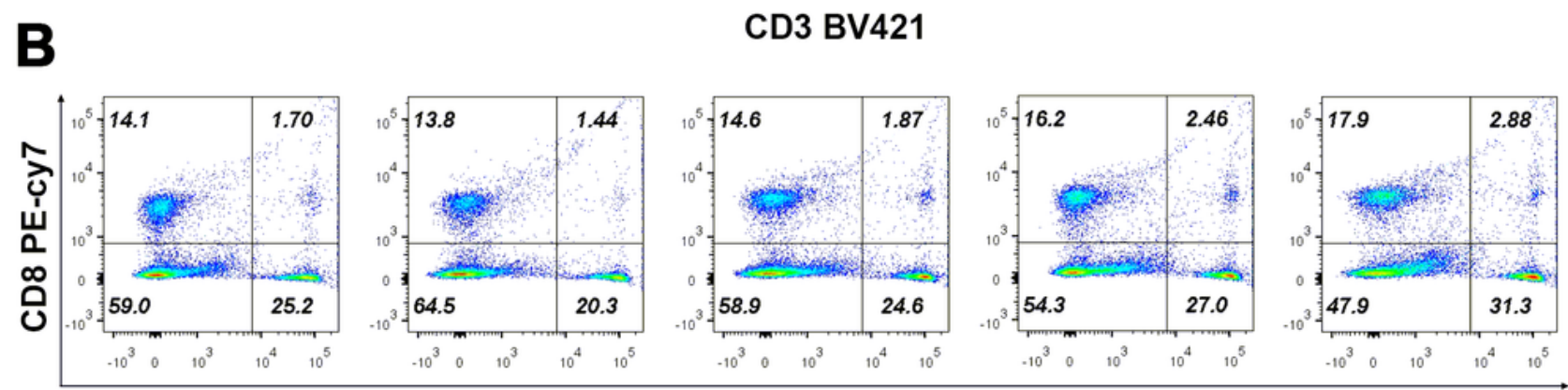

CD4 PE

C

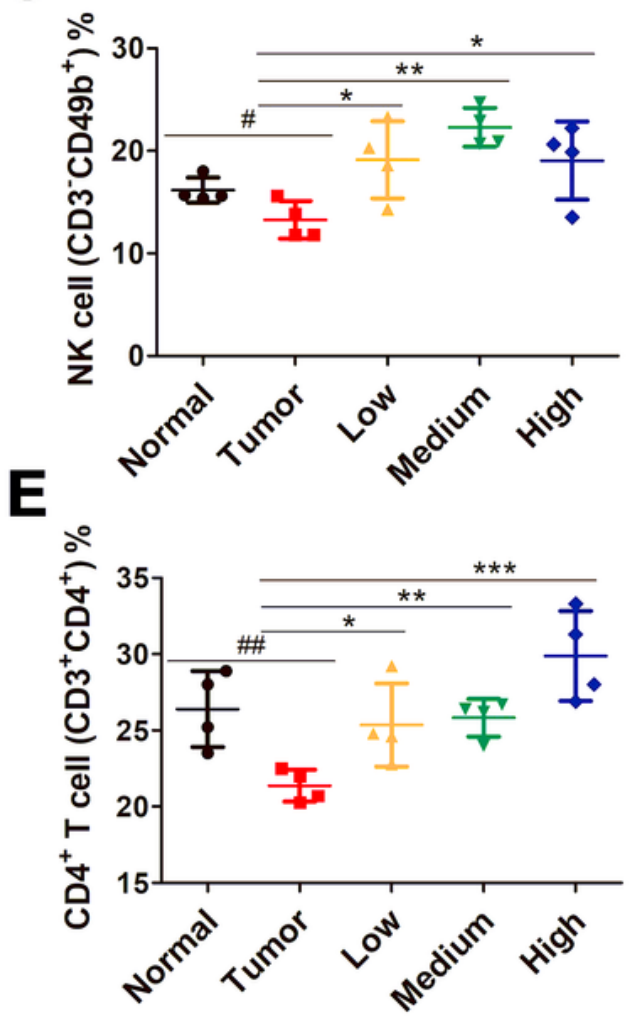

D
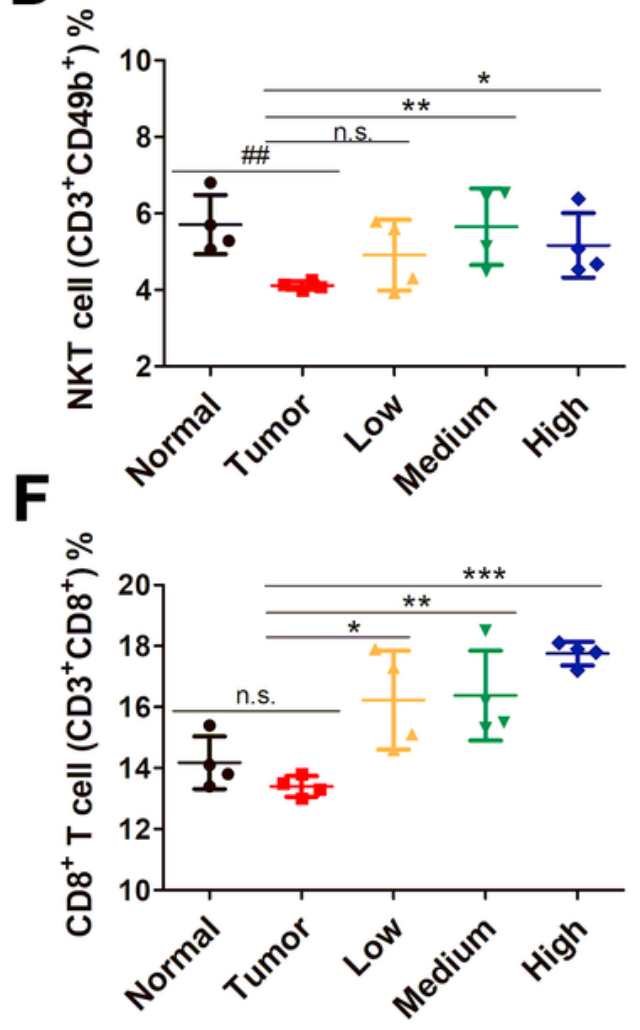

Figure 7

Icariside I improves proportions of multiple lymphocytes in the peripheral blood of tumor-bearing mice.

(A) Representative flow cytometric analysis images of NK cells (CD3-CD49b+) and NKT cells (CD3+CD49b+). (B) Representative flow cytometric analysis images of CD4+ T cells (CD3+CD4+) and CD8+ T cells (CD3+CD8+). Corresponding quantification of NK (C), NKT (D) cells and CD4+ (E), CD8+ (F) 
T cells. Values were represented the means $\pm S D(n=4)$. \#p $<0.05, \# \# p<0.01$ and \#\#\#p<0.001 versus Normal group; ${ }^{*} p<0.05,{ }^{*} p<0.01$ and ${ }^{\star * \star} \mathrm{p}<0.001$ versus Tumor group.

\section{Supplementary Files}

This is a list of supplementary files associated with this preprint. Click to download.

- SupplementalmaterialsSubmission.docx

- SupplementalmaterialsSubmission.docx 